IMMUNOMETABOLISM

\section{Fast tracking immunity}

Three recent studies in Cell explore how the nutritional stress associated with fasting affects the immune system.

Collins et al. found that mice subjected to dietary restriction (DR; $50 \%$ reduced food intake) for 3-4 weeks had fewer antigenexperienced $\mathrm{T}$ cells, $\mathrm{B}$ cells and natural killer cells in the periphery. Closer examination showed that memory $\mathrm{T}$ cell numbers increased in the bone marrow (BM) during $\mathrm{DR}$, whereas other lymphocyte populations were preserved in number but did not increase. DR promoted the accumulation of central and effector memory $\mathrm{T}$ cells in the BM rather than enhancing their homeostatic proliferation; indeed, these cells were less proliferative during DR.

Glucocorticoids (GCs) are known to be crucial for regulating energy balance. The authors found GC levels increased in the circulation during DR and this was necessary for memory $\mathrm{T}$ cell redistribution to the BM. High GC concentrations promote $\mathrm{T}$ cell death, and memory T cells that accumulated in BM during DR showed less cell death and higher expression of anti-apoptotic BCL-2.

Whole-tissue RNA sequencing and further experiments indicated that the bone marrow microenvironment is profoundly remodelled during DR. In particular, enhanced signalling through the CXCL12-CXCR4 and S1P-S1P1R axes and increased adipogenesis in the BM seemed to promote memory $\mathrm{T}$ cell accumulation following $\mathrm{DR}$. Memory T cells that accumulated in BM during DR also showed lower metabolic activity and reduced mTOR signalling.

Remarkably, the authors found that mice placed on DR in between a primary and secondary bacterial challenge showed an enhanced memory $\mathrm{T}$ cell response to the infection. Similarly, DR improved antitumour memory $\mathrm{T}$ cell responses and mouse survival in a model of melanoma. Therefore, DR is able to enhance memory $\mathrm{T}$ cell responses in the context of infection and tumour immunity.
The study by Nagai et al. examined the effects of fasting on young mice ( $\sim 6$ weeks old). The authors focused on the gut mucosa and found that Peyer's patches (PPs) were markedly reduced in size and cellularity when mice were deprived of food for 36 hours. This was associated with increased apoptosis of proliferating B cells and IgA class-switched B cells, but not naive B cell apoptosis. Upon refeeding, PPs became predominantly filled with naive $B$ cells, suggesting that these cells were temporarily leaving the PPs during fasting.

Indeed, further studies showed that naive B cells shuttle between PPs and the BM during fasting and refeeding cycles, and this was associated with fluctuating expression of the B cell chemoattractant CXCL13 in PPs and BM. Experiments using a lymph nodederived stromal cell line suggested that glycolysis is necessary for CXCL13 expression in stromal cells.

The authors subsequently subjected mice to fasting and refeeding for four cycles and at the end of each refeeding cycle, the mice were orally immunized with ovalbumin (OVA) and cholera toxin. Compared with control mice, the fasted mice showed markedly reduced levels of OVA-specific fecal IgA as well as lower serum levels of specific IgA, IgG and IgM. Fasting also impaired specific IgA responses during Salmonella infection and exacerbated diarrhoeal disease in a model in which $\operatorname{Ig} A$ and $\operatorname{Ig} G$ are protective factors.

Jordan et al. found that fasting reduces the pool of circulating monocytes in mice and in healthy humans. Closer analysis of mice showed that fasting also reduced pro-inflammatory $\mathrm{LY} 6 \mathrm{C}^{\text {hi }}$ monocytes in peripheral tissues. The authors found that LY6C $\mathrm{C}^{\text {hi }}$ monocytes accumulate in the $\mathrm{BM}$ during fasting, suggesting that fasting may block monocyte egress from the BM.

Blockade of glycolysis had a similar effect to fasting in reducing circulating monocyte numbers, and activation of the low-energy sensor AMPK was sufficient to prevent monocyte egress into the blood. Intriguingly, fasting did not block monocyte egress in

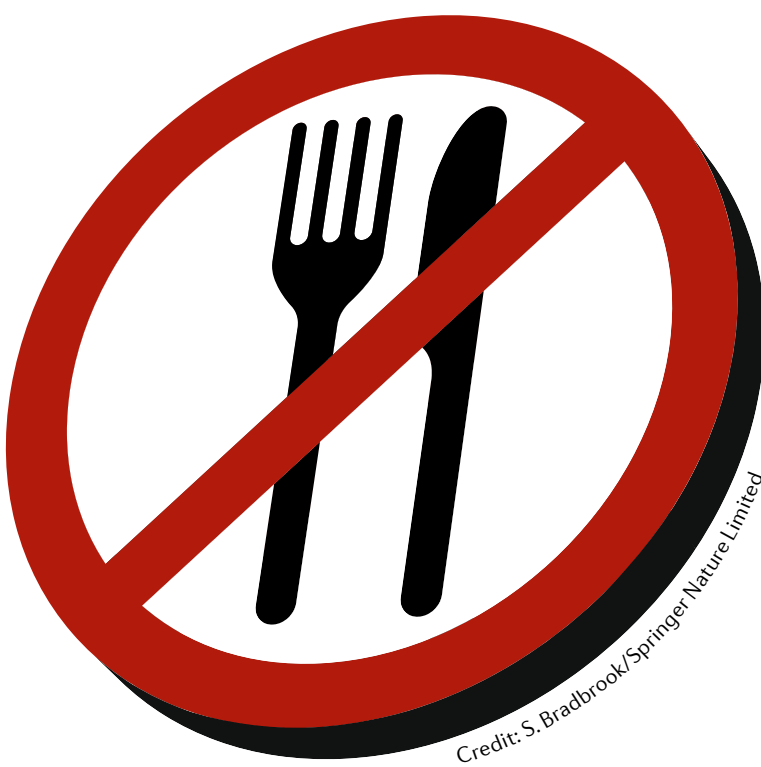

mice with a hepatocyte-specific loss of AMPK or PPARa (a downstream target of AMPK).

Levels of the monocyte chemoattractant CCL2 were reduced in the plasma of fasting humans and mice, but fasting-induced loss of CCL2 was not observed in PPAR $\alpha$-deficient mice. BM production of CCL2 correlated most closely with peripheral monocyte numbers and the authors found that at least 33 liver-derived factors that regulate CCL2 production are modulated during fasting. Therefore, energy sensing in the liver can regulate the peripheral monocyte pool via effects on distal CCL2 production.

Monocytes from fasting mice also showed reduced metabolic activity and, importantly, the authors found that fasting improved disease outcomes in models of chronic inflammation but, strikingly, did not compromise monocyte mobilization during infection.

Collectively, these studies show that fasting can modulate immune responses in the context of infection, inflammatory disease and cancer. As such, carefully designed dietary interventions could be used to support immune function in various clinical settings.

Yvonne Bordon

to support

immune

function in various clinical settings

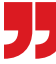

ORIGINAL ARTICLES Collins, N. et al. The bone marrow protects and optimizes immunological memory during dietary restriction. Cell 178 , 1088-1101 (2019) | Nagai, M. et al. Fastingrefeeding impacts immune cell dynamics and mucosal immune responses. Cell 178, 1072-1087 (2019) | Jordan, S. et al. Dietary intake regulates the circulating inflammatory monocyte pool. Cell 178, 1102-1114 (2019) 